\title{
DISEÑO DE UN MODELO DE INTERVENCIÓN PSICOSOCIAL PARA LA POBLACIÓN USUARIA DEL CENTRO DE ATENCIÓN PSICOSOCIAL - CAP DE LA UNAD CEAD FLORENCIA, CON BASE EN LA CARACTERIZACIÓN DE SU PERFIL INDIVIDUAL, FAMILIAR Y SOCIAL
}

\author{
Juan Pablo Castaño ${ }^{52}$
}

RESUMEN

Conocedores del alto costo psicosocial que genera en la sociedad civil el problema del desplazamiento forzado a causa del conflicto armado, la presente investigación tiene como objetivo diseñar un Modelo de Intervención Psicosocial para la población en situación de desplazamiento y vulnerabilidad del Departamento del Caquetá. Se ha propuesto desarrollar la investigación en tres fases: la fase inicial, que consiste en caracterizar a la población objeto del proyecto desde el punto de vista psicosocial; la segunda fase implica el diseño del Modelo de Intervención Psicosocial, tomando como ejes estructurales las dimensiones individual, familiar y social de los consultantes; y la fase final hace referencia a la implementación y ejecución del modelo desde el Centro de Atención Psicosocial CAP.

La investigación es de tipo cualitativa, emplea como muestra 144 consultantes de los 156 que tuvieron asesoría psicosocial durante el periodo mayo-diciembre de 2009, y utiliza como instrumento en su primera fase la información consignada en las historias clínicas de los consultantes. El proyecto actualmente se encuentra finalizando su etapa de caracterización de la población objetivo, y busca, además de incrementar el bienestar y la calidad de vida de la población desplazada y vulnerable desde el punto de vista psicosocial, fortalecer la proyección social comunitaria de la UNAD en el contexto local y regional.

Palabras clave: modelo psicosocial, desplazamiento, vulnerabilidad.

\begin{abstract}
Aware of the high psychosocial costs the society generates the problem of forced displacement due to the armed conflict; this research aims to design a Psychosocial Intervention Model for the population in situation of displacement and vulnerability of the Department of Caquetá. It is proposed to develop the investigation in three phases: the initial phase, which is to characterize the target population of the project from a psychosocial perspective, the second phase involves the design of Psychosocial Intervention Model, on the main structural individual dimensions, family and social consultants and the final stage refers to the implementation and execution of the model from the Psychosocial Care Center CAP.
\end{abstract}

52 Grupo de Investigación: Ingeniería Humana, Código del Grupo en Colciencias: COL0072139, Nombre del semillero: Arquitectos de realidades humanas, Línea de investigación: Contextos socioculturales, procesos psicosociales, comunitarios y psicología social comunitaria. Red de Investigación: Desarrollo Humano, Convivencia y Productividad. UNAD. 
The research is qualitative, 144 used as a sample of the 156 consultants who had psychosocial counseling during the period from May to December 2009 and used as an instrument in its first phase the information in the medical records of the consultants. The project is currently finished in its stage of characterization of the target population, and also seeks to increase the wellbeing and quality of life of the displaced population and vulnerable from the psychological point of view, strengthen community-based social outreach in UNAD local and regional context.

Key words: psychosocial model, displacement, vulnerability.

\section{INTRODUCCIÓN}

El fenómeno del desplazamiento masivo a causa del conflicto armado es una realidad que desde hace más de cuatro décadas afecta de manera directa e indirecta a gran parte de la población en el país, y sus principales consecuencias siempre han desembocado en muerte, pobreza y sufrimiento. Esta violencia, que en la mayoría de los casos tiene bases políticas, se ha definido como la que se ejerce como medio de lucha político-social, ya sea con el fin de mantener, modificar, sustituir, destruir o reprimir a un grupo humano con identidad dentro de la sociedad por su afinidad social, política, gremial, étnica, racial, religiosa, cultural o ideológica, esté o no organizada. ${ }^{53}$

No ajeno a esta problemática, el Departamento del Caquetá es uno de los lugares donde el conflicto armado se ha enraizado con mayor intensidad, lo cual ha generado desplazamientos masivos en casi todos los 16 municipios que lo componen. Según cifras de la oficina de Acción Social Regional Caquetá (informe del 2009), en el departamento existen alrededor de 95.526 personas registradas como desplazadas en el RUPD (Registro Único de Población Desplazada), y con ellas vienen a cuesta también todas las consecuencias físicas, psicológicas, familiares y sociales. Frente a esta realidad social que se presenta a nivel local y regional, las instituciones del orden gubernamental y no gubernamental (Acción social, UAO (Universidad Autónoma de Occidente), Fiscalía General de la Nación, Alcaldía de Florencia, ICBF (Instituto Colombiano de Bienestar Familiar), Personería, Procuraduría, Defensoría del Pueblo, UNAD, Pastoral Social, Médicos Sin Fronteras, Organización Internacional para las Migraciones, Comité Internacional de la Cruz Roja, etc.) hacen sus mayores esfuerzos por atender a la población afectada intentando satisfacer sus necesidades más apremiantes y contribuyendo para que su proceso de adaptación a su nueva realidad sea más rápido y efectivo.

Por lo tanto, la presente investigación busca fortalecer la oferta institucional que de manera particular brinda la UNAD a la población desplazada y vulnerable del departamento a través de los servicios psicosociales del Centro de Atención Psicosocial CAP, ubicado en el CEAD Florencia. Para ello, desea diseñar un modelo de atención que permita atender las necesidades de la población objetivo desde el punto de vista individual, familiar y social, tomando como base la caracterización de la población atendida en el CAP durante el año 2009. De esta manera,

$\overline{53 \text { Noche \& Niebla, } 1998 .}$ 
pretende además que los servicios de extensión que ofrece la universidad a la población beneficiaria estén estructurados bajo la rigurosidad y aplicación del método científico, con lo cual se brinde un producto o servicio de excelente calidad que contribuya por un lado al desarrollo de la investigación en la academia, y por el otro, brinde el beneficio social para lo cual fue creado.

\section{OBJETIVO GENERAL}

Diseñar un modelo de intervención psicosocial para la población en situación de desplazamiento usuaria del Centro de Atención Psicosocial - CAP, de la UNAD CEAD Florencia, con base en la caracterización de su perfil individual, familiar y social.

\section{OBJETIVOS ESPECÍFICOS}

Caracterizar desde el punto de vista psicosocial a la población desplazada usuaria del CAP, tomando como base para el levantamiento de la información los datos registrados en las historias clínicas.

Orientar el diseño del modelo de intervención psicosocial tomando como referentes las dimensiones individual, familiar y social de los consultantes.

Implementar el modelo de intervención psicosocial como estrategia para mejorar los estándares de calidad en la atención que brinda el CAP a sus consultantes.

Promover la esencia social comunitaria de la UNAD a través del CAP como una estrategia de extensión universitaria.

\section{METODOLOGÍA}

La investigación es de tipo cualitativo, en la medida que pretende profundizar en la comprensión de la realidad psicosocial de la población en situación de desplazamiento atendida en el Centro de Atención Psicosocial CAP, de la UNAD CEAD Florencia.

La población total es de 156 consultantes atendidos en el periodo mayo-diciembre de 2009, quienes tienen la característica de ser población en situación tanto de desplazamiento como de vulnerabilidad. Sin embargo, se toma como muestra 85 participantes, sobre el criterio de ser desplazados certificados, es decir, que se encuentran en la base de datos RUPD de Acción Social. Cada uno de ellos fue contactado telefónicamente para obtener su autorización verbal de emplear sus datos e información en el estudio.

Se ha propuesto desarrollar la investigación en tres fases: la fase inicial, que consiste en caracterizar a la población objetivo del proyecto desde el punto de vista psicosocial; la segunda fase implica el diseño del Modelo de Intervención Psicosocial, tomando como ejes estructurales las dimensiones individual, familiar y social de los consultantes; y la fase final 
hace referencia a la implementación y ejecución del modelo desde el Centro de Atención Psicosocial CAP.

\section{A. FASE I: Caracterización de la población objetivo}

En esta fase se realizó la revisión de las historias clínicas de los consultantes que asistieron al CAP durante el periodo 15 de mayo a 15 de diciembre de 2009. Para ello se tomó como base la información registrada en la historia clínica del consultante desde su dimensión individual tales como edad, sexo, nivel educativo, estrato (ver anexo núm. 1); información de su dimensión familiar relacionada con tipo de familia, ciclo evolutivo de sus miembros, número de integrantes de la familia (ver anexo núm. 2); y la información relacionada con su dimensión social tales como ocupación, nivel de ingresos, factores de riesgo psicosocial y síntomas asociados a su motivo de consulta (ver anexo núm. 3). Esta etapa se encuentra ya culminada, es decir, se cuentan con los resultados de la caracterización con los cuales se procede a dar inicio a la fase dos.

\section{B. FASE II: Diseño del Modelo de Intervención Psicosocial}

Esta epata consiste en diseñar el Modelo de Intervención Psicosocial sobre la base de una revisión teórica exhaustiva que permita identificar los diferentes modelos de atención e intervención que existen desde la Psicología Social Comunitaria y que son empleados en la atención de la población en situación de desplazamiento. Dicha revisión bibliográfica se realiza a la luz de las 3 dimensiones estructurales del modelo que son: lo individual, lo familiar y lo social, al igual que toma para su análisis y diseño, los resultados obtenidos en la caracterización psicosocial de la población.

\section{FASE III: Implementación y ejecución del modelo desde el Centro de Atención Psicosocial CAP de la UnAD CEAD Florencia}

El modelo entra a operar dentro del procedimiento que se lleva en el CAP del CEAD Florencia, el cual atiende a los usuarios de lunes a viernes en horario de 8:00 am a 12:00 $\mathrm{m}$ y de 2:00 pm a 6:00 pm. Esto implica que cada usuario atendido a partir de la entrada en vigencia del modelo, será intervenido desde sus dimensiones individual, familiar y social con el propósito de encontrar alternativas cada vez más efectivas que satisfagan sus necesidades en estas dimensiones, y con ello pueda encontrar mayor bienestar y mejoramiento de las condiciones de vida en las cuales se encuentra. 


\section{RESULTADOS PARCIALES}

Los resultados parciales obtenidos en la primera etapa del proyecto son los que caracterizan la población en situación de desplazamiento desde sus dimensiones individual, familiar y social, y que han consultado en el CAP de la UNAD CEAD Florencia. Los resultados que a continuación se presentan, contienen las gráficas relacionadas con cada una de dichas dimensiones.

\section{A. DIMENSIÓN INDIVIDUAL (Anexo núm. 1)}

\section{SEXO}

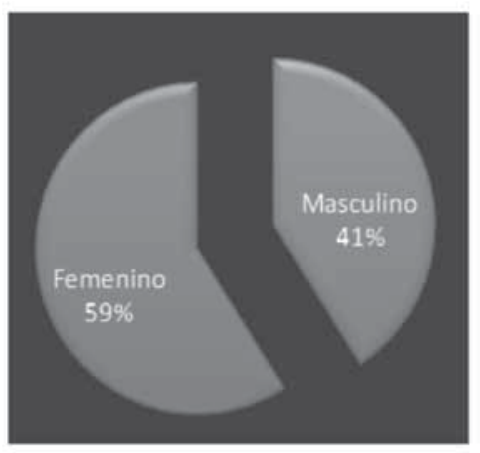

3. NIVEL DE ESCOLARIDAD

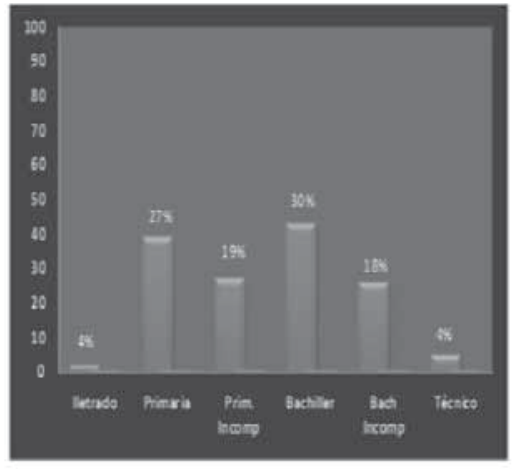

2. ESTADO CIVIL

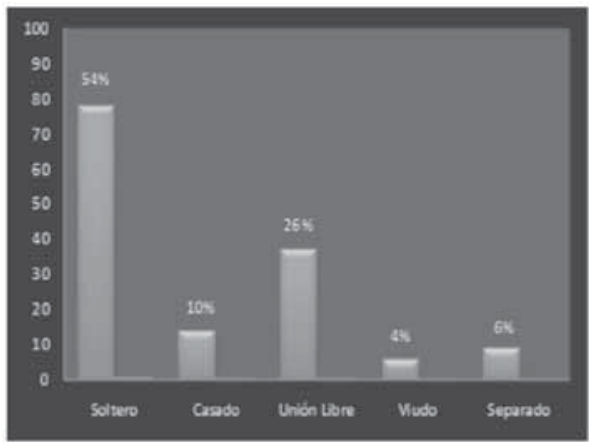

4. ESTRATO

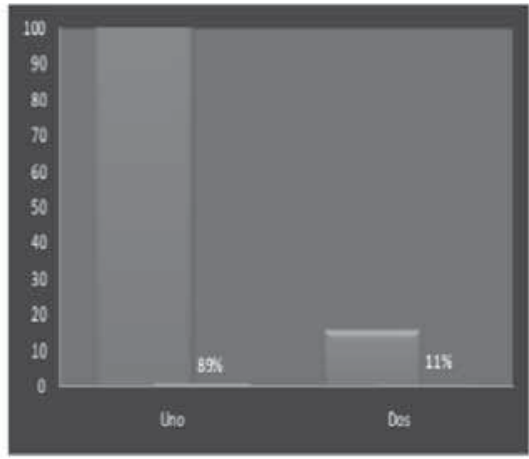


Revista de InVestigaciones UNAD

\section{B. DIMENSIÓN FAMILIAR (Anexo núm. 2)}

1. TIPO DE FAMILIA

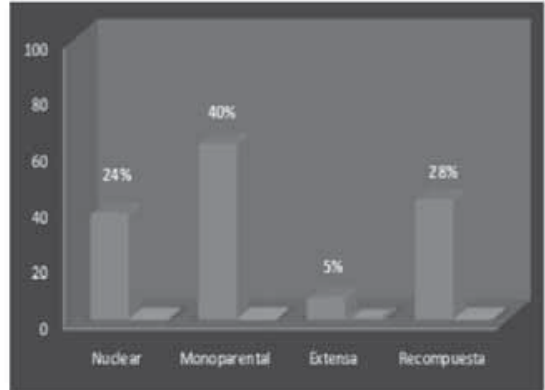

3. NÚMERO DE INTEGRANTES

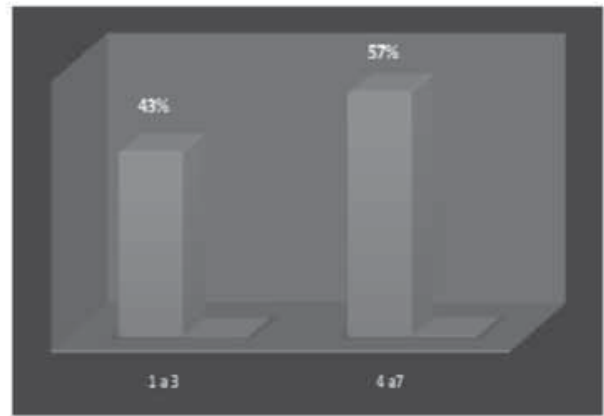

C. DIMENSIÓN SOCIAL (Anexo núm. 3)

1. OCUPACIÓN

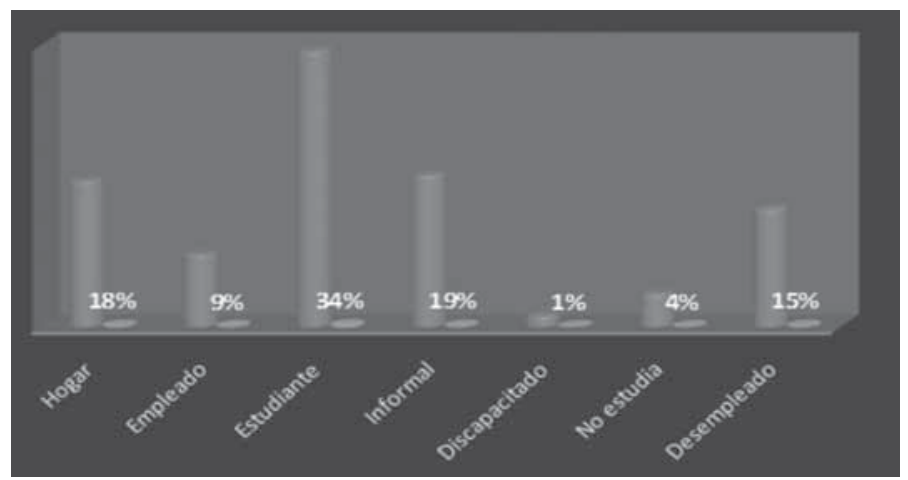

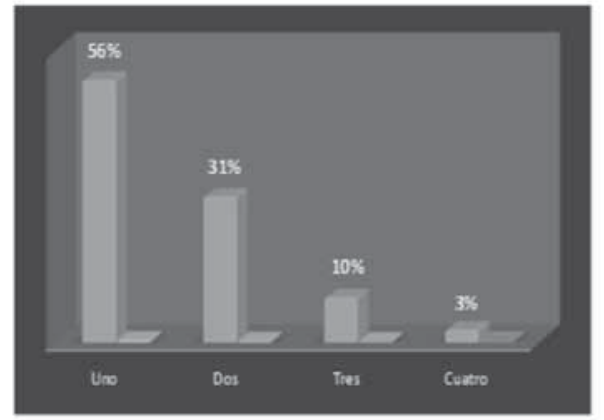

4. NÚM. DE APORTANTES EN LA FLIA
2. CICLO EVOLUTIVO

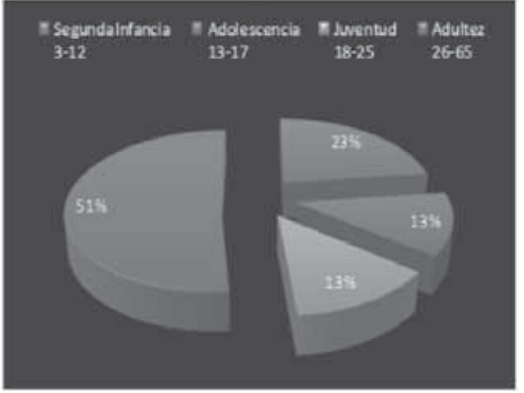
A 
2. TENENCIA DE LA VIVIENDA

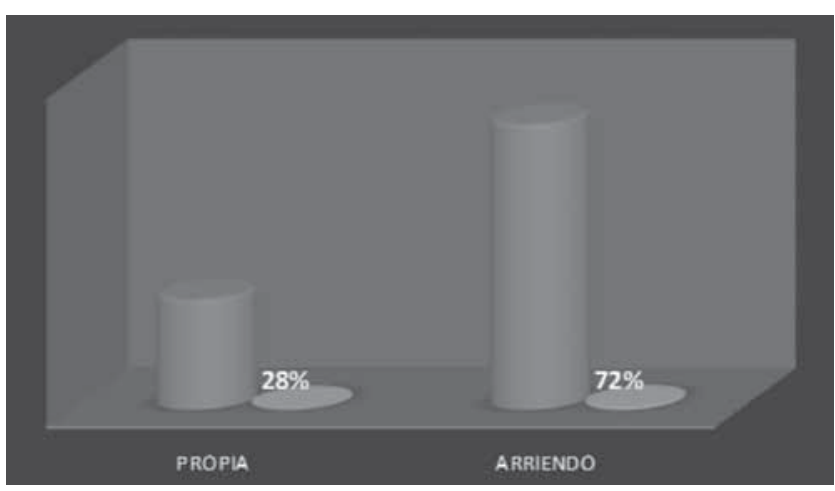

3. FACTORES DE RIESGO

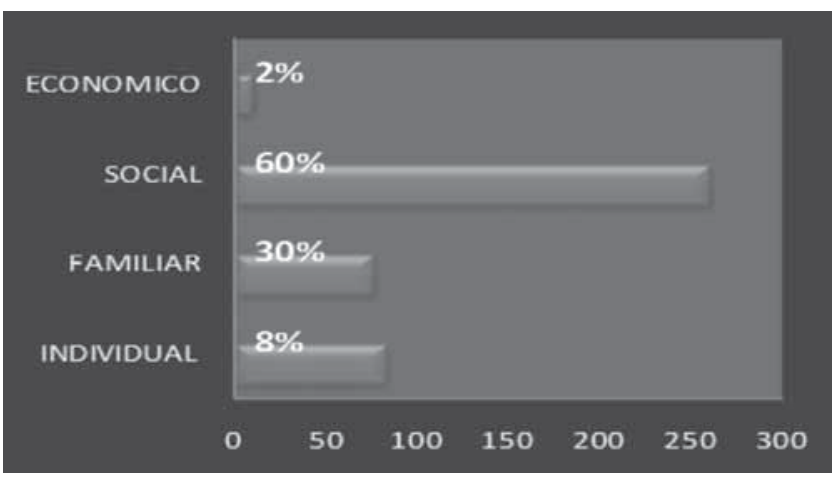

4. SIGNOS Y SÍNTOMAS

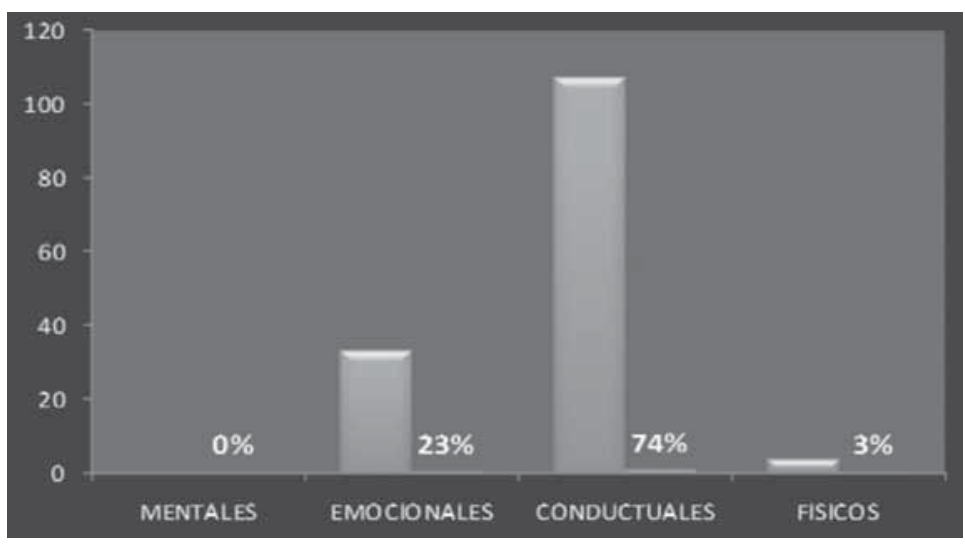




\section{DISCUSIÓN}

En un primer momento, se presentan los aspectos o variables generales relacionadas con la población atendida. En este sentido, se pueden identificar los siguientes resultados:

Es más frecuente que las mujeres sean las que consulten (59\%), en lugar de los hombres (41\%). Esto se explica en parte porque el desplazamiento afecta la dinámica familiar, los roles y el ejercicio del liderazgo en el hombre y la mujer de distintas maneras. El hombre en la ciudad se siente inhabilitado en su actuar, está acostumbrado a trabajar la tierra o estar en el campo, sus exigencias por lo regular son físicas. Las mujeres por naturaleza tienden a ser más sociables, abiertas al diálogo, y por la misma situación producto del desplazamiento, toman la iniciativa de pedir ayuda, tarea que el hombre le cuesta realizar. Máxime si para el hombre, pedir ayuda es un acto de "debilidad".

En un 54\% las personas que consultan se encuentran en condición de estar solteras(os) o solteras(os) con hijos, y en menor proporción (36\%) viven en pareja. Sólo el $4 \%$ refieren estar viudos y el 6\% dicen estar separados. Dentro de la población soltera, esta condición se presenta en parte debido a las malas experiencias afectivas del pasado, lo cual es motivo suficiente para continuar así, y no les interesa iniciar por el momento una nueva relación.

Es preocupante el poco desarrollo que tiene el aspecto educativo en la población usuaria. Sólo el 30\% ha terminado sus estudios de bachillerato, y aproximadamente el $65 \%$ tienen la condición de ser iletrados, haber culminado la primaria y tener cursado la primaria y el bachillerato incompleto. En parte puede ser porque la mayoría de la población en situación de desplazamiento vienen del sector rural, y la educación no está en los primeros lugares de prioridad. Diferentes estudios han demostrado la relación directa que hay entre el bajo nivel educativo y la violencia y el maltrato intrafamiliar, las deficientes pautas de crianza y la baja asertividad para resolver los conflictos.

El 89\% de la población se encuentran dentro del estrato socioeconómico uno (1), lo cual es reflejo de la falta de condiciones y carencias de todo tipo que acompaña a esta población. Sólo el $11 \%$ tiene un poco de mejores condiciones que el grupo anterior, pues se encuentran en el nivel socioeconómico dos (2). Asociado a su condición de desplazamiento se suma el alto nivel de vulnerabilidad que padece esta población, y las implicaciones que esto trae para sus dimensiones bio-sico-socio-familiares.

En cuanto a la ocupación que desempeñan los consultantes, el mayor grupo (39\%) que ha asistido a consulta son estudiantes, lo cual es un indicador que refleja que muchos de los problemas están relacionados con los hijos de la población desplazada en cualquiera de sus etapas del ciclo evolutivo en que se encuentran. Un 28\% laboran de manera formal o informal, como mecanismo de generación de ingresos para satisfacer sus necesidades básicas; sin embargo este factor debe ser analizado más cuidadosamente en la medida que permite inferir también si la población desplazada está considerando dentro de sus prioridades el emplearse o trabajar de alguna manera, o si por el contrario prefieren esperar el auxilio para sobrevivir de 
lo que el Estado les brinda, lo cual es una actitud poco saludable desde todo punto de vista. El 18\% están dedicados a realizar actividades en el hogar y el 15\% son desempleados, es decir, no están empleando su capacidad de trabajo para generar ingresos para si mismo o para su familia.

De manera importante las cifras presentan a un $60 \%$ de los consultantes que viven como familia monoparental, es decir, uno de los padres e hijos viviendo bajo un mismo techo. Por otra parte, son casi similares el número de personas que han decido asumir la responsabilidad de su familia bajo la figura nuclear, es decir, mamá, papá e hijos $(20 \%)$ y las que han decidido darse una segunda oportunidad bajo la forma de familia reconstituida (20\%). Estos resultados evidencian las tendencias sociales que persisten y que modelan los estereotipos de conformación de una familia de hoy.

Dentro del ciclo evolutivo de una persona, el $51 \%$ de los consultantes se encuentran en edad adulta ( 26 a 50 años), lo cual puede inferirse que una gran parte de esta generación de personas adultas de una u otra manera le ha tocado experimentar las secuelas del conflicto y la guerra que vive el país. Sin embargo, otro dato preocupante es que en un $24 \%$, los niños de 3 a 12 años han sido víctimas del conflicto armado de una u otra manera, lo cual confirma la afirmación que los niños de este país han aprendido a vivir con la guerra a cuestas, y de paso con todas sus caras y manifestaciones.

Lo anterior se evidencia con los signos y síntomas que presentan los consultantes a la hora de acudir a las sesiones. Los mayores síntomas están asociados con trastornos conductuales tales como agresividad y ansiedad. Luego siguen los síntomas emocionales relacionados con el llanto, la depresión, la preocupación excesiva y el temor.

En cuanto a los factores de riesgos psicosociales que afectan a la población consultante y que son los generadores de los síntomas que estos manifiestan, se encuentra en primer término los factores de riesgo sociales $(60 \%)$ tales como muerte violenta, desplazamiento, secuestro, reclutamiento, convivir con actores armados y exposición al conflicto. En segundo aspecto aparece el factor de riesgo familiar (30\%) con situaciones asociadas a problemas de pareja, dificultades en la red de apoyo primaria, violencia intrafamiliar y maltrato infantil. Y finalmente, los factores individuales en menor proporción $8 \%$ (consumo de sustancias psicoactivas, entre otras variables). Estos factores son las principales fuentes generadoras de estrés y sintomatología de la población usuaria de los servicios psicosociales del proyecto.

Los resultados anteriores permiten contar con una primera aproximación a la realidad psicosocio-familiar de la población desplazada, y son un punto de análisis inicial para orientar el diseño del Modelo de Intervención Psicosocial en el CAP.

\section{CONCLUSIONES}

La realidad psicosocial de la población en situación de desplazamiento es tan compleja y profunda que se requiere analizarla desde diferentes ópticas para llegar a tener una pequeña comprensión de sus múltiples dinamismos y formas de expresión. 
La paz, el bienestar social, el desarrollo humano y la calidad de vida no dependen solamente de la resolución del conflicto armado actual. Son muchas las formas de violencia que aquejan a la sociedad de este país, y poco es lo que se hace como intervención en las demás esferas generadoras de violencia social.

La educación es un pilar fundamental para vivir en paz; sin embargo, como lo demuestra la historia pasada y reciente, no ha sido ni será a corto plazo, prioridad para los gobiernos de turno, a pesar que todos sepan de su vital importancia.

La población atendida hasta el momento demuestra que primero están sus necesidades de subsistencia (alimentación, techo y abrigo) que las necesidades relacionadas con su satisfacción interior y su salud psicosocial. Un sufrimiento por la pérdida de un ser querido se puede llevar a cuestas incluso por muchos años, pero el hambre o el sueño es algo que no da espera, y es lo primero que se debe atender.

Lo anterior puede explicar en parte la conducta de deserción tan frecuente que se presenta en la población atendida hasta el momento. El número de sesiones promedio por persona es de 1.5 , lo cual indica que la persona con la descarga emocional, el llanto, el poder expresar sus sentimientos, el escuchar otro punto de vista sobre su realidad, entre otros aspectos, descansa, siente un relativo bienestar y con ello vuelve a enfrentar su mundo cotidiano.

En vista del alto costo emocional que trae el conflicto armado para la población civil, es fundamental continuar con la oferta institucional que se realiza desde el CAP en la medida que constituye una alternativa de solución o de afrontamiento a la compleja realidad que integra la vida de las personas en situación de desplazamiento.

\section{REFERENCIAS BIBLIOGRÁFICAS}

ACCIÓN SOCIAL REGIONAL CAQUETÁ, diciembre de 2009. Informe de Gestión.

CODHES. (1995), Derechos Humanos y Desplazamiento interno en Colombia:

INVESTIGACIÓN SOBRE DERECHOS HUMANOS Y DESPLAZAMIENTO INTERNO EN COLOMBIA. Bogotá. Conferencia Episcopal de Colombia.

EXODO. (1998), Boletín sobre desplazamiento interno en Colombia Núm. 7. Bogotá. Arte y Fotolito.

FEIJOO, N. (1994), Construcción de una escala para medir estrés. Revista avances en psicología clínica latinoamericana. 12, pp. 47-62.

GARCÍA, M. (1999), Los desplazados por la violencia en Colombia. Con su dolor sin rumbo. Revista Universitas Humanística. Ene-Jun. Núm. 47. Pontificia Universidad Javeriana. Bogotá. 
NOCHE Y NIEBLA NÚM. 38, julio diciembre 1998: El desplazamiento en Colombia: Una realidad a cuestas.

SÁNCHEZ, R. Y JARAMILLO, L. (1999), Impacto del desplazamiento sobre la salud mental. Revista Universitas Humanística. Ene-Jun. Núm. 47. Pontificia Universidad Javeriana. Bogotá.

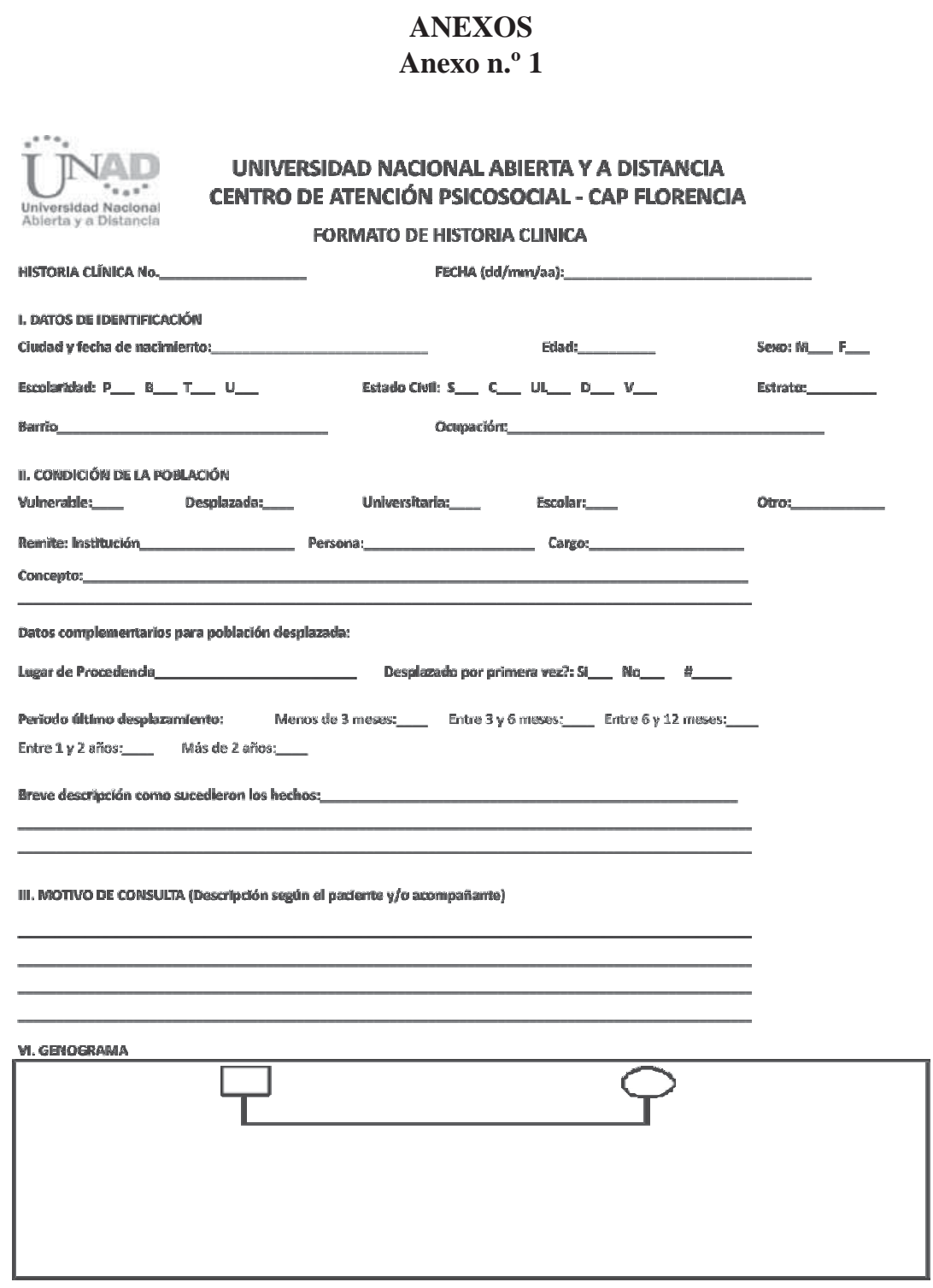

